\title{
BoNuS: Development and Use of a Radial TPC using Cylindrical GEMs*
}

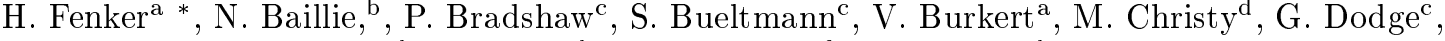 \\ D. Dutta ${ }^{\mathrm{e}}$, R. Ent $^{\mathrm{a}}$, J. Evans ${ }^{\mathrm{b}}$, R. Fersch ${ }^{\mathrm{b}}$, K. Giovanettif ${ }^{\mathrm{f}}$, K. Griffioen ${ }^{\mathrm{b}}$, M. Ispiryan ${ }^{\mathrm{g}}$, C. Jayalath ${ }^{\mathrm{d}}$,

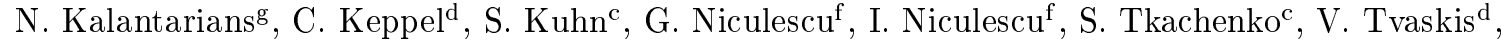 \\ J. Zhang ${ }^{\mathrm{C}}$
}

a Thomas Jefferson National Accelerator Facility, Newport News, VA 23606

${ }^{\mathrm{b}}$ College of William and Mary, Williamsburg, VA

${ }^{\mathrm{c}}$ Old Dominion University, Norfolk, VA

${ }^{\mathrm{d}}$ Hampton University, Hampton, VA

e Tri-Universitites Nuclear Lab, Durham, NC

${ }^{\mathrm{f}}$ James Madison University, Harrisonburg, VA

${ }^{\mathrm{g}}$ University of Houston, Houston, TX

To provide new access to information about nucleon structure from electron-neutron interactions, a specialized system of target and detector was developed at Jefferson Lab. It allows identification and measurement of spectator protons produced in $e^{-} d \rightarrow e^{-} p_{s} X$ scattering events. The detector is a radial time-projection chamber optimized for the acceptance of low-momentum protons. Gas gain is provided by three cascaded curved Gas Electron Multipliers (GEMs), the first application of GEMs in any configuration other than flat.

\section{Introduction}

Ever since the GEM was first described[1] it was apparent that it might serve very naturally as the gas-gain element of a time projection chamber (TPC)[2]. Subsequently, several groups[3,4] have demonstrated this technique. GEMs allow an almost arbitrary readout electrode pattern (strips, pads, chevrons, etc.) which can be chosen to suit the particular needs of a given application. Previously, electrode configurations were constrained by the orientations of the wires used for gas gain. With GEMs, no wire-orientation artifacts exist in the resulting data because the GEM serves as a uniform amplification surface having no preferred orientation.

Thus far, all of these electrode patterns have been formed on planar surfaces and the GEMs

\footnotetext{
* Notice: Authored by The Southeastern Universities Research Association, Inc. under U.S. DOE Contract No. DE-AC05-84ER40150 . The U.S. Government retains a non-exclusive, paid-up, irrevocable, world-wide license to publish or reproduce this manuscript for U.S. Government purposes.
}

were also flat. As noted early[5], however, there is no reason that GEMs could not be formed into curved surfaces to mate with similarly curved charge-collection electrodes. In the present work we report on the development, construction, and operation in a nuclear physics experiment of a radial time projection chamber (RTPC) in which the GEMs and readout surfaces are cylindrical. The use of GEMs allowed us to form the detector in a shape that was natural for the experiment and to eliminate the relatively massive structural components that would have been necessary to maintain wire tension.

\section{Physics Motivation}

Over the history of nuclear and particle physics much has been learned about the structure of the proton through scattering experiments using proton targets in the form of hydrogen nuclei. Equally fundamental scattering data on neutrons is much more difficult to obtain, however, since free neutrons do not exist in ordinary matter. 
The closest approximation to a free neutron in matter is the partner of a proton in a deuterium nucleus. Estimates of neutron scattering properties may be found by noting the differences between deuteron and proton scattering data, and applying model-dependent corrections for nuclear binding effects.

An example of this sort of analysis is shown in Fig. 1. It shows the recoil mass distributions obtained under similar conditions when electrons are scattered from a hydrogen target and from a deuterium target. While one would expect similar richness of the resonance spectrum in both protons and neutrons, it is apparent from the figure that the experimental technique does not provide a clear picture of the neutron resonance spectrum: the effects of nuclear binding and final-state interactions smear the observed mass distribution. Similar difficulties exist in experiments probing neutron form factors and structure functions.

In the absence of a truly free neutron target, it is possible to improve the experimental situation if individual beam-deuteron scattering events can be identified as having involved primarily the neutron as the target particle. The situation gets even better if those events with minimal finalstate interactions and only weak nuclear-binding effects can be selected. A technique that achieves these goals is spectator-proton tagging, which is straightforward to apply in deuterium bubble chamber experiments [9], and has also been implemented in a few counter experiments [10].

In the spectator-proton method, one identifies events as involving a neutron target by observing the presence of the essentially unperturbed initial nuclear proton in the final state of the interaction. Particularly, the presence of a final-state proton moving backwards relative to the beam particle signifies a very likely neutron-target event with minimal interaction between the proton and other final-state particles. Measurement of this proton's momentum constrains the initial state (Fermi momentum) of the neutron at the time of the collision, leading to a correct determination of the kinematics of the beam-neutron interaction. This technique is the basis of the BoNuS (Barely off-shell Nucleon Structure) experiment, E03-012 at Jefferson Lab, which is the
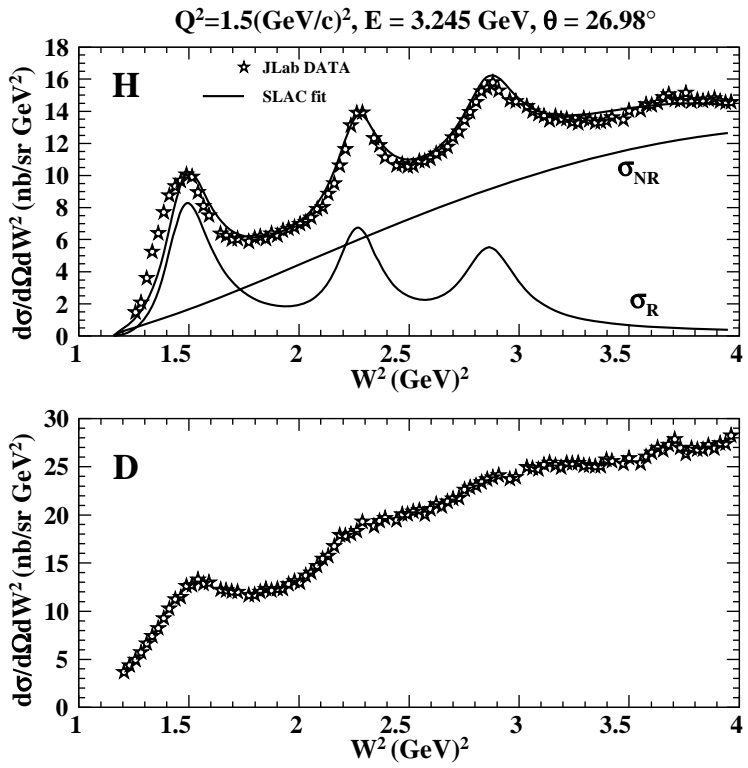

Figure 1. Inclusive resonance electroproduction cross sections from JLab at $Q^{2}=1.5 \mathrm{GeV}^{2}$ [6]. Cross sections are shown as a function of invariant recoil mass squared for hydrogen (top) and deuterium (bottom) targets at matched kinematics. The hydrogen spectrum is plotted with global, resonant, and non-resonant fits $[7,8]$.

beginning of an experimental program to study electron-neutron scattering[11]. The piece of experimental hardware at the core of this experiment is a time projection chamber designed to detect and measure spectator protons. It surrounds the beamline and a high-pressure gas target, and sits within the CEBAF Large Acceptance Spectrometer (CLAS)[12] in Hall-B of Jefferson Lab.

\section{Detector Concept}

\subsection{Physics Considerations in the Detec- tor Design}

To outline the required characteristics of the spectator proton detector for the BoNuS experiment, it is necessary to know the momentum and angular distributions of the protons, the rates and types of background particles that will penetrate 
the detector, and the event rate that must be accommodated. For this purpose, the Fermi motion of the nucleons within a deuterium nucleus is adequately described by the Hulthen wavefunction[13]. The angular distribution of the nucleons is isotropic in the lab frame, although the experimentally observed distribution will be modified by the energy-dependence of the scattering cross section. The nucleon momentum distribution is shown in Fig. 2, where it can be seen that momenta in the range of tens of $\mathrm{MeV} / \mathrm{c}$ are favored. The low energy spectators are also the ones least likely to have been boosted by final state interactions. Thus it is crucial that the detector accept protons with the lowest possible energy over a wide angular range.

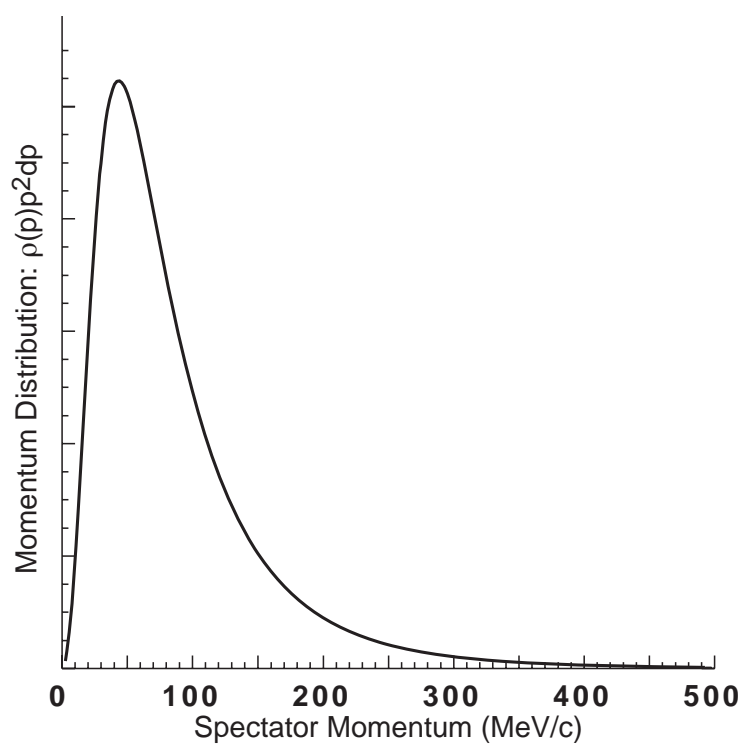

Figure 2. Deuterium Nucleon Momentum Distribution as modeled by the Hulthen wavefunction.

To perform the experiment as efficiently as possible, it was desired to fully exploit the data acquisition capacity of the existing CLAS systems by taking data at a rate of at least $2 \mathrm{kHz}$. This leads to a design luminosity of about
$10^{34} \mathrm{~cm}^{-2} \mathrm{~s}^{-1}$, which can be achieved with a $6 \mathrm{GeV} / \mathrm{c}$ electron beam current of $200 \mathrm{nA}$ striking a $12.6 \mathrm{mg} / \mathrm{cm}^{2}$ deuterium target $(20 \mathrm{~cm}$ of deuterium gas at 7 bar pressure). The experiment proposal shows that some 3 million e-n backwards-spectator events are needed to form a compelling statistical sample of collisions for resonance production and form-factor studies, which can be collected in about 30 days of running at the above rate.

With such a beam and target, interactions with atomic electrons produce a huge flux of Moller electrons. This background would completely flood the spectator detector if it passed through the tracking region. One standard technique for avoiding this is to provide a strong magnetic field throughout the target region, with the field lines parallel to the beam, so that Moller electrons are trapped in a small cone extending downstream from the target. A massive absorber further downstream then stops the bulk of this background. Of course, the detector sensitive region can begin only outside this high-background zone. Fortuitously, this same magnetic field provides momentum analysis of spectator protons.

The ionization energy loss of $100 \mathrm{MeV} / \mathrm{c}$ protons is about 25 times that of minimum-ionizing particles. This ratio increases as the proton momentum decreases. Very little material is required to stop such heavily-ionizing particles. This consideration leads naturally to the choice of a TPC to detect the spectator protons because of the very low density of such detectors. It also leads to the choice of a helium-based mixture for the sensitive gas as well as the use of the thinnest possible entrance windows so that low momentum protons can enter and pass through the tracking volume. The low density requirement also impacts the design of the experimental target, as the protons must have a reasonable probability of exiting the target volume.

It is convenient to make use of the large difference in the densities of the ionization trails between the slow protons we wish to measure and the lightly-ionizing background particles we wish to suppress. This density difference is helpful during analysis to distinguish even forward-going spectator protons from other collision products. 
As will be discussed below, it also allows one to set the gas gain and electronic threshold such that the detector is not sensitive to particles other than those likely to be spectator protons.

\subsection{Experimental Constraints and Con- ceptual Design}

The chosen detector is a TPC which surrounds a long, thin, gas target, and which would fit inside an available 4 Tesla solenoid magnet [14]. The length of the target, and therefore the length of the TPC, was influenced both by the need to obtain the required luminosity without stopping the spectator protons in the target itself, as well as the requirement to correlate interactions observed in the spectator detector with interactions detected in the CLAS tracking system.

The target is a $250 \mathrm{~mm}$ long $6 \mathrm{~mm}$ dia. straw made of spiral-wound $50 \mu \mathrm{m}$ thick Kapton ${ }^{\circledR}[15]$. It is filled with target gas (normally $\mathrm{D}_{2}$, but also $\mathrm{H}_{2}$ or He for calibration data) at pressure up to 7 bar. The TPC active volume is $200 \mathrm{~mm}$ long. The target extends $50 \mathrm{~mm}$ downstream of the TPC to reduce the acceptance for particles scattered from its downstream window $(30 \mu \mathrm{m} \mathrm{Al})$. The upstream target window is coincident with the upstream end of the TPC, but triggers from interactions on this window and in the first $50 \mathrm{~mm}$ of target are suppressed by a metal cylinder surrounding this portion of the target. Interactions in this region are of less interest because there is no acceptance for backwards spectators originating here.

The TPC and the on-chamber electronics must fit within the 220-mm bore of the magnet. This TPC system is mounted on the end of a $3-\mathrm{m}$ long tube through which the beamline vacuum pipe as well as all utility and signal lines are routed. At the upstream end, the tube is fixed to a large rail-mounted cart which allows the detector to be inserted into the bore of the magnet and surveyed into place. Fig. 3 shows a diagram of this structure and the location of the BoNuS detector within a cross section of CLAS.

In a conventional cylindrical TPC the ion trails are forced toward either the upstream or downstream end cap where the gas-gain components and readout are located. This makes the end caps somewhat massive because of the electronics and cables, but the position resolution often benefits by having the ionization electrons drift along magnetic field lines parallel to the cylinder axis.

In the BoNuS experiment, secondary particles from the events, notably the scattered highenergy electrons, travel predominantly forward and must pass through the downstream end cap of the TPC before they enter the CLAS tracking, particle identification, and calorimetry systems. Clearly it is advantageous to minimize the end cap density. Further, the solenoid magnet available for use has a length to diameter ratio less than unity leading to significant divergence of the field lines within the TPC drift volume. This divergence would cause large portions of tracks in an axial-drift TPC to be lost as the ionization electrons, drifting along the field lines, would impact chamber boundaries away from the end caps.

For these reasons it was decided to employ the radial drift technique in the BoNuS TPC. Ionization electrons move radially outward, roughly perpendicular to the magnetic field, and arrive on the inside of the outer cylindrical surface of the TPC. With the radial extent of the detector being smaller than its length, this also shortens the drift path and reduces the time required to clear a track out of the sensitive volume. An example of an RTPC already exists in the STAR experiment [16] at Brookhaven National Lab. Readout electrodes, electronics, and cables can reside on the curved surface of the cylinder leaving the downstream end cap free of such massive components. While ion trails drifting across the magnetic field lines will follow a curved path, this complication can be dealt with as the data are analyzed.

With this basic design in mind, it remained to decide how to provide gas gain and gating structures in the detector. STAR, for example, used modules consisting of anode wires and readout strips placed at intervals around the drift volume. Most TPCs with readout on the end caps use wires for gain, along with various configurations of gating grids to prevent positive ions produced in the avalanches from drifting back into the sensitive volume. In more recent work, inves- 


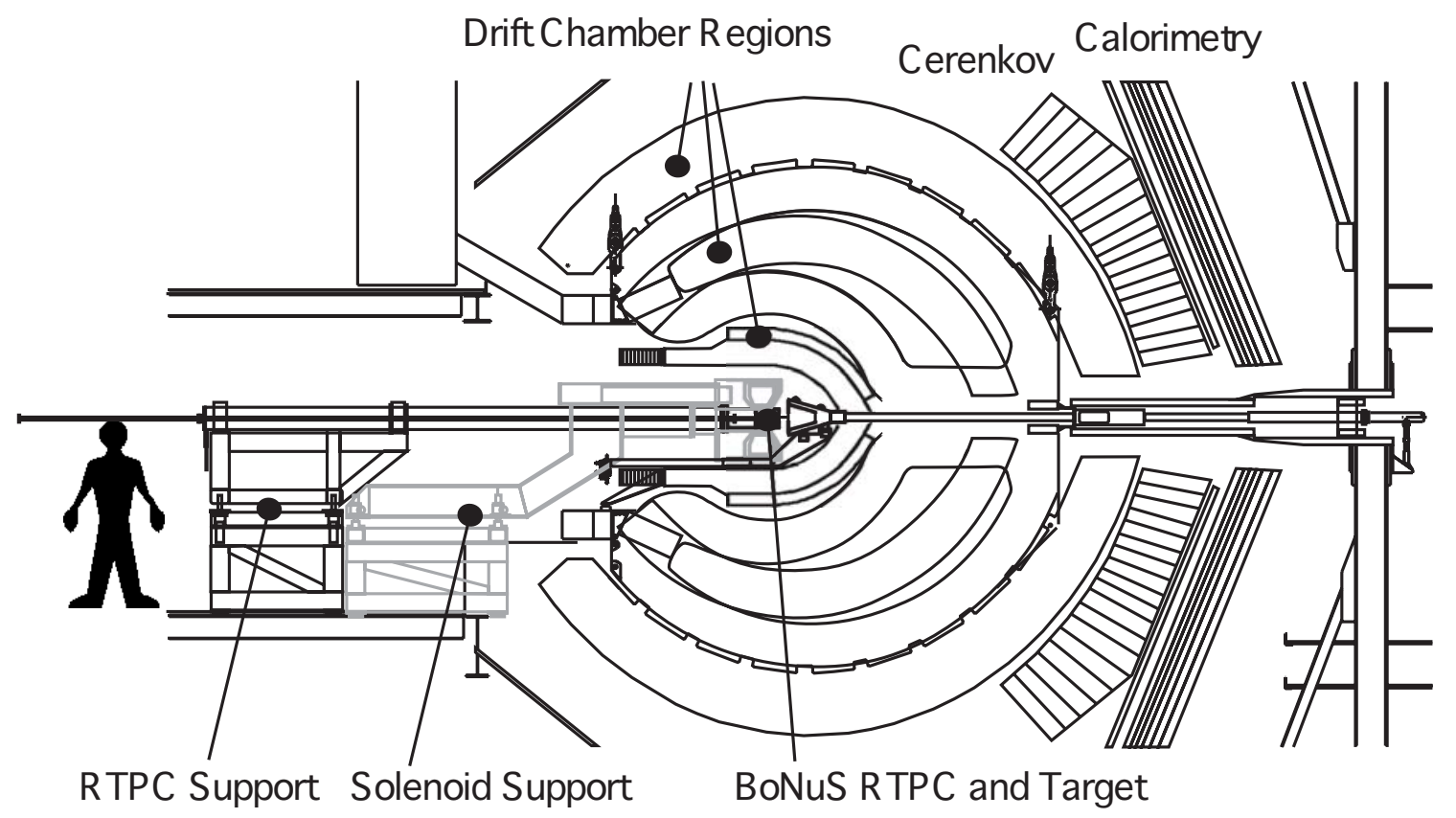

Figure 3. Cross Section of the BoNuS RTPC Location within CLAS. The RTPC is on the end of its support structure and positioned at the center of the CLAS system, along the beamline. The target (not shown) is surrounded by the RTPC. The solenoid magnet surrounds the RTPC.

tigators have been using micro-pattern gas detectors, including GEMs[17]. GEMs were suggested by Sauli as a means to improve the performance of TPCs in general[2], and, for the BoNuS detector in particular, he stated that the RTPC with cylindrical GEM readout would be "the simple and natural solution to the problem" [18]. Prior to the work described in this report, however, nobody had tested GEMs in any configuration other than planar, although it was anticipated that there was no fundamental reason why they would not work[5].

\section{Prototype Studies of Cylindrical GEM RTPCs}

To determine the feasibility of using curved GEMs in the BoNuS RTPC, several prototype detectors were built and tested. The goal of this effort was to build a small RTPC which would expose problems to be encountered with construc- tion and operation of the full size BoNuS detector. Thus these GEMs should have the same curvature as those needed for BoNuS and they should be supplied by a manufacturer that could also produce the custom-designed GEMs needed in the production detector. Further, the mechanical components and readout configuration should be similar to what would be needed in the final design.

\subsection{Flat GEM TPC Prototype}

The first step was to construct a simple flat GEM TPC to learn how to operate even the simplest form of such a device and to demonstrate that it could be used to track and distinguish minimum-ionizing particles and heavily-ionizing protons. A cross section of the resulting detector is shown in Fig. 4. From top to bottom it is composed of an aluminized-mylar entrance window, an aluminized-mylar cathode, 19.1-mm tracking region, three GEMs, and a printed-circuit board 
(PCB) patterned with 2.5-mm wide strips attached to a connector. The tracking volume was usually filled with a mixture of $20 \% \mathrm{CO}_{2}$ and $80 \%$ Ar at atmospheric pressure.

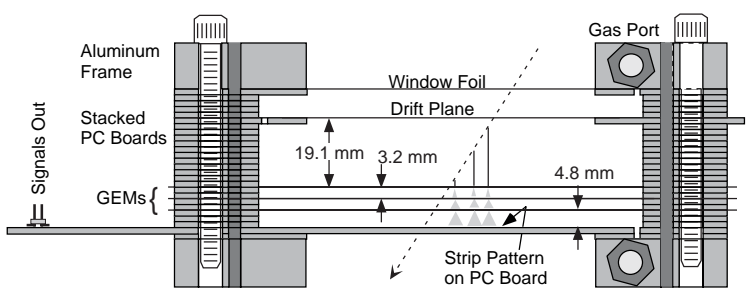

Figure 4. Cross Section Diagram of the $10 \mathrm{~cm} \times$ $10 \mathrm{~cm}$ Flat TPC.

GEMs used in this module were produced by $3 \mathrm{M}$ [19]. They were in the standard $10 \mathrm{~cm} \times 10 \mathrm{~cm}$ format originally produced by CERN, and were stretched and attached to FR4 PCB frames $1.59 \mathrm{~mm}$ thick. As shown in the figure, the entire detector assembly was composed of a stack of several of these frames compressed between two aluminum plates. Gas ports in the plates mated with appropriate holes and passages in the window frame and readout-board to provide gas circulation.

The most significant challenges in the construction of this prototype involved management of the high-voltage systems. Initial attempts to make the entrance window (at ground potential) and the drift electrode (at up to $-5 \mathrm{kV}$ ) from a single piece of $\mathrm{Cu}$-Kapton- $\mathrm{Cu}$ laminate were unsuccessful. Using standard printed-circuit techniques, the conductor was etched away from the outer $10 \mathrm{~mm}$ of both sides of a $120 \mathrm{~mm} \times$ $120 \mathrm{~mm}$ sheet of material. The remaining electrodes were charged by a high-voltage power supply. All samples thus prepared eventually failed when the $50 \mu \mathrm{m}$ thick Kapton ${ }^{\circledR}$ broke down at some point along the edge of the copper cladding. Failures in the center were unusual, reflecting the very high dielectric strength of Kapton ${ }^{\circledR}(\approx$ $175 \mathrm{kV} / \mathrm{mm})[15]$.
The successful design of the cathode and window separated these two functions. Each one is made of a sheet of mylar $6.4 \mu \mathrm{m}$ thick which has been coated on both sides with a $35 \mathrm{~nm}$ thick layer of aluminum. This material is commonly used as a thermal barrier in cryogenic systems. The window is spaced $8 \mathrm{~mm}$ away from the cathode and the volume between them is flushed with the same gas used in the tracking volume. This gap is sufficient to support the high potential difference $(\sim 5 \mathrm{kV})$ across it. Although the gap takes up additional space, the two-component design has the advantage of presenting much less mass than one double-clad layer of insulator having the same dielectric strength.

High voltage to all of the detectors described in this report was provided by a simple divider circuit and two power supplies as indicated in Fig. 5. This circuit is a variation of an earlier design[20] which provides nearly simultaneous shutdown of all high voltages in the event of a fault, as well as separate adjustment of the TPC drift field and the potentials affecting gas gain in the GEMs. Both power supplies are referenced to ground, so the voltage across the tracking region is determined by the difference between the two supply set-points. It is important to choose resistors whose values will not drift either because of the applied voltages (low VCR such as the Ohmite Slim-Mox ${ }^{\circledR}$ [21] series) or because they get hot (sufficient power rating). In cases where the GEM electrodes were subdivided on one side of the GEM, a separate $1 \mathrm{M} \Omega$ current limiting resistor was provided for each segment (only one of these resistors is shown for each GEM in the figure).

Initially the data acquisition system consisted of only eight channels of waveform digitizer (LeCroy 2262 [22]), so the single-coordinate readout was appropriate to enable detection of particle tracks over a volume large enough to obtain a few cosmic ray tracks per minute $(19.1 \mathrm{~mm} \times$ $20 \mathrm{~mm} \times 100 \mathrm{~mm})$. Signal pulses from each strip were amplified by a relatively fast ( 5 ns risetime) transimpedance preamplifier (gain $\approx 10 \mathrm{mV} / \mu \mathrm{A}$ ) from which the differential output was converted to a negative-going analog signal appropriate for driving the $50 \Omega$ inputs of the digitizers. 


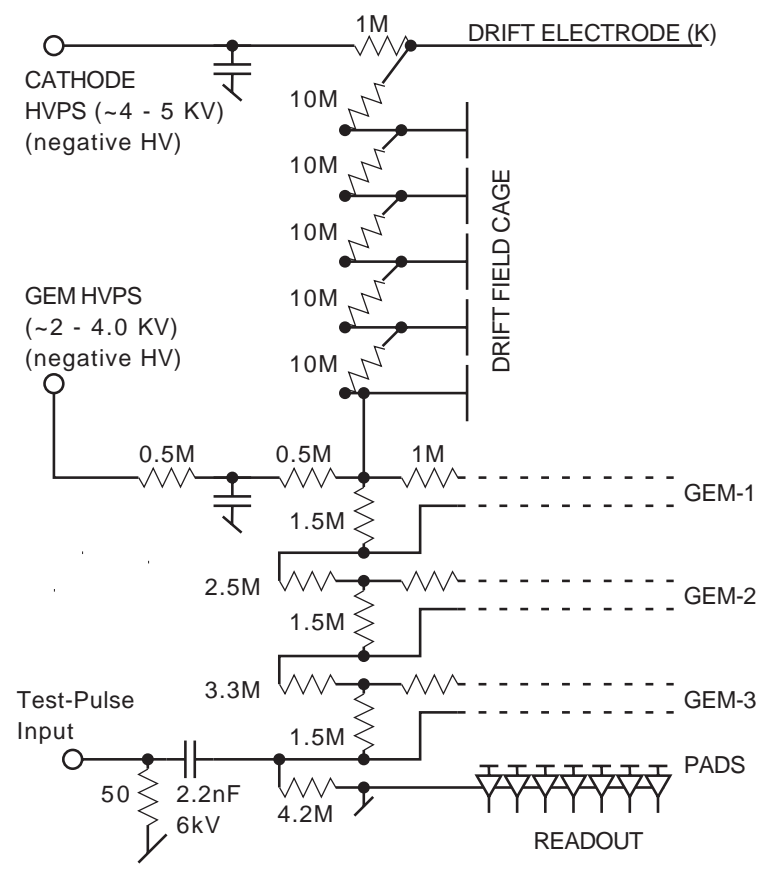

Figure 5. Two-Channel High Voltage Divider Circuit used in all of the TPCs described in this report. Resistor values shown are only typical. Actual values chosen for use varied.

A ${ }^{55} \mathrm{Fe}$ source was used to generate signals in the detector to allow optimization of the GEM and intermediate electric fields and to measure the gas gain. Some of the source data will be described below. However, the ultimate purpose of this prototype was to demonstrate the fundamental tracking capabilities of such a device. Fig. 6 shows graphically the signals produced by a cosmic ray and $\mathrm{a} \sim 5 \mathrm{MeV}$ proton $^{2}$ from the TUNL tandem accelerator[23]. Triggering of the readout for cosmic rays was provided by the coincidence of a pair of scintillators, while for protons (which cannot penetrate the scintillator paddles) the trigger was derived from the appearance of

\footnotetext{
${ }^{2}$ The tandem energy was approximately $10 \mathrm{MeV}$. The lower energy protons entering the detector were obtained by scattering the primary beam through $90^{\circ}$ off of a goldplated tungsten wire. Proton energy was further degraded by passage through air and a plastic absorber.
}

a signal in the TPC itself. The dense ionization trails left by protons $(\sim 600$ pairs $/ \mathrm{mm}$ in $\mathrm{ArCO}_{2}$ ) are easily distinguished from the sparse trails caused by minimum-ionizing cosmic rays $(\sim 25$ times less than the slow protons).

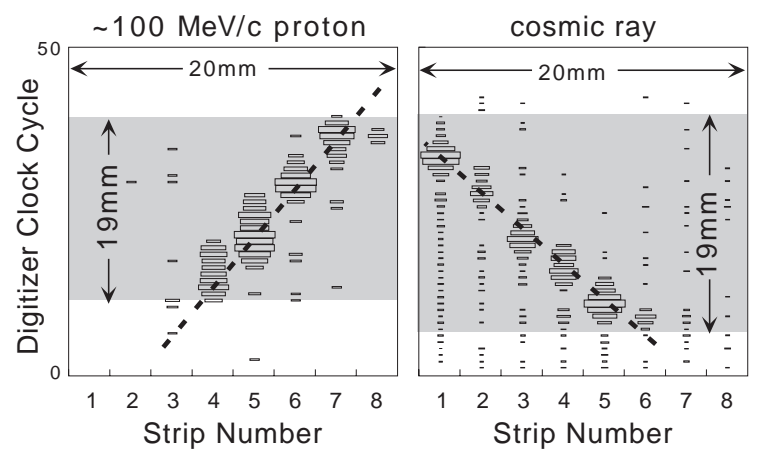

Figure 6. Event displays from the flat prototype TPC when traversed by a low-energy proton (left) and a cosmic ray (right). The size of each box indicates the signal pulse-height at each sampling interval for each strip. It was necessary to increase the GEM gain (high voltage) in order to track cosmic rays. The shaded portions of the displays indicate the drift-time window corresponding to the physical chamber volume. The drift velocities were different when these two events were recorded.

\subsection{Curved Prototype}

The design of the prototype curved-geometry detector is essentially an extension of that of the flat prototype. Open frames made of Ultem ${ }^{\circledR}[24]$ support the GEMs, the cathode, the thin inner window, and the charge-collection electrodes (padboard). The frames are formed in a curved shape, however, as though they had been cut out of a cylindrical surface. Diagrams of this prototype module are shown in Fig. 7.

It was important to build this prototype in such a way that the GEMs and other components would have the same curvature as the elements of 
the final BoNuS detector. The cathode has radius $40 \mathrm{~mm}$, the GEMs are at radii of 60,63 , and $66 \mathrm{~mm}$, and the padboard inner radius is $69 \mathrm{~mm}$. (The ultimate design of the production detector uses a $30 \mathrm{~mm}$ radius cathode.) The inner window of the curved prototype is placed at $r=25.4 \mathrm{~mm}$. Because the available GEMs had an active area of $10 \mathrm{~cm} \times 10 \mathrm{~cm}$, the above GEM radii constrained the prototype to cover about $105^{\circ}$ around the axis and $10 \mathrm{~cm}$ along it.

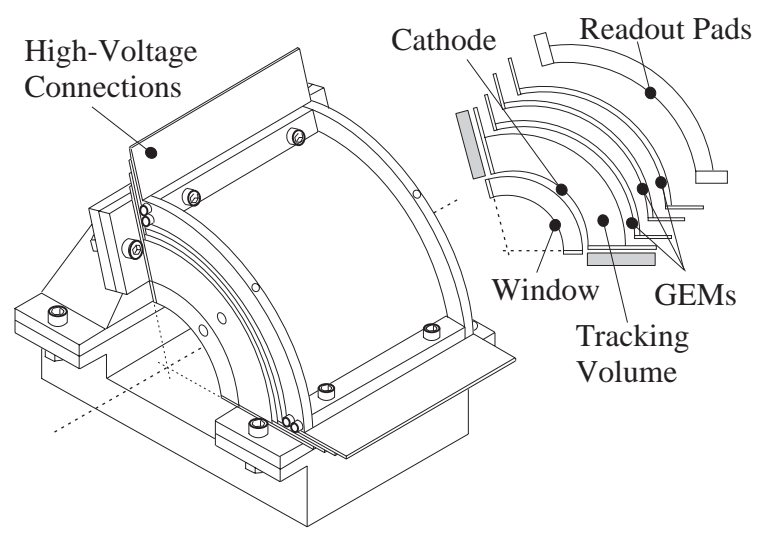

Figure 7. Perspective View and Exploded Cross Section of the Prototype Curved RTPC

To provide attachment points for the high voltage connections, flat PCBs are used for the straight portions of the frames. Their surfaces are oriented parallel to radii of the cylindrical assembly. The frame end-pieces are circular arcs whose thicknesses determine the spacing between the components they support. Each sub-assembly consists of two arcs and two flat boards. These pieces were held in the correct relative orientation on a custom fixture and then bonded together by epoxy(TRA-BOND 2129[25]). When the detector is assembled, the cylindrical arcs nest within one-another and the flat PCBs lie on top of oneanother.

The cathode and window materials were attached to their respective frames using minor variations on the following basic GEM mounting procedure. Untrimmed foils $(15 \mathrm{~cm} \times 15 \mathrm{~cm})$ were aligned and laid across a semi-cylindrical mandrel fabricated with the correct radius for that particular foil. Placement of the GEM was done by hand and did not put it under tension, but simply made it lie smoothly against the mandrel. It was fixed in position by adhesive tape. The corresponding frame subassembly was then coated with a thin layer of epoxy on its inner surfaces and placed on the GEM, using mechanical guides built into the assembly fixtures to assure proper orientation. The frame was clamped in place against the GEM and mandrel and the epoxy was allowed to cure. Fig. 8 shows the first curved GEM at this stage of preparation. Excess material was trimmed away from the outside of the assembly, taking care to protect the high-voltage leads from each side of the GEM. These were carefully folded and glued to lie against the flat PCBs where metal traces bring the high-voltage connections to the outside for attachment to the external circuit.

Forming the outer surface of the RTPC is the padboard. This is a PCB with pad electrodes on one side and a standard commercial connector pattern on the other. Each pad is connected by traces and a closed via to one of the connector nodes. The board was made using a thin FR-4 substrate so that it could be rolled and then held at the desired radius by its frame. The board used in this prototype is $\sim 380 \mu \mathrm{m}$ thick, and was preformed by clamping it around a $100 \mathrm{~mm}$ dia. tube while baking it at about $120^{\circ} \mathrm{C}$ for a few hours. The basic pad shape is a rectangle with dimensions approximately $4 \mathrm{~mm} \times 5 \mathrm{~mm}$, the $5 \mathrm{~mm}$ side being parallel to the cylinder axis. A geometry with each axial row of pads offset from its neighboring rows in a quasi-randomized brickwork arrangement is the final preferred design (see Fig. 11). It reduces the likelihood of a track which projects along a single row of pads, which would lead to poorer position resolution.

Operating gas is introduced to the chamber through a port in the arc between the inner window and the cathode. A port and tube in the arc on the opposite ring carry the gas from this inner gap to the drift region. From here, the gas flows through the GEMs and exits the chamber 


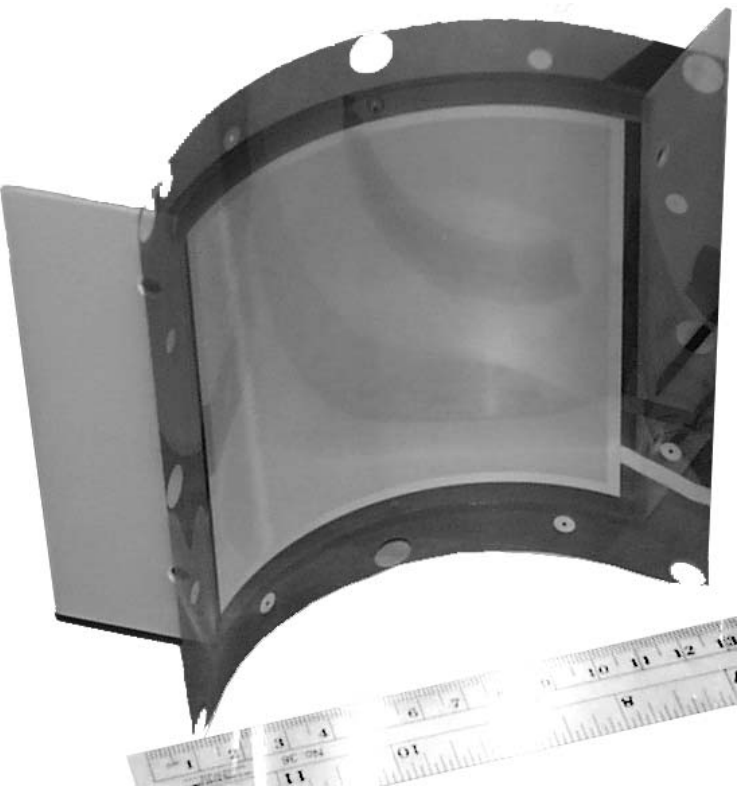

Figure 8. First Curved GEM after attachment to its frame. The excess material has not yet been cut away.

through a hole in the padboard to which an exhaust tube is attached. This flow path was chosen so that the pressure gradient across the cathode material would be radially outward, causing the cathode to form the desired cylindrical shape. The same effect reinforces the natural tendency of the GEMs to assume such a shape, although the pressure drop across one of these GEMs at a flow rate of $\sim 1$ liter/minute is negligible. Electrostatic attraction between the GEMs and the padboard is more significant, and also makes each GEM tend toward a cylindrical shape. Although the inner window is bowed inward toward the cylinder axis by gas pressure, this has no impact on the operation of the detector.

\subsection{Performance of Curved GEMs}

This prototype detector appears to be the first application of curved GEMs, so it is appropriate to describe its performance in comparison to that of the planar detector. The curved-GEM prototype detector was turned on and immediately began to exhibit operating characteristics very similar to its flat predecessor. No unusual difficulties were encountered. It was subjected to tests using cosmic rays, radioactive sources, and $\sim 100 \mathrm{MeV} / \mathrm{c}$ protons.

Pulse-height spectra from a ${ }^{55} \mathrm{Fe} \mathrm{X}$-ray source were obtained using both the curved-GEM RTPC and the planar-GEM test chamber. Two of these spectra are provided for comparison in Fig. 9. These data were taken using different GEM designs, very different chamber designs, and slightly different electronics. While quantitative comparisons would be unreliable, it is clear from the figure that GEMs retain their basic functionality after being formed into cylinders. The gas gain needed to drive the electronics chosen for the readout (see below) is in the range $0.5 \times 10^{5}-$ $1.0 \times 10^{5}$ for minimum-ionizing tracks, a value readily achieved with both planar and curved triple-GEM configurations.

Other researchers [3,27] reported variations in the gas-gain of GEMs as a function of the length of time that high voltage had been applied ("charge-up") and as a function of the flux of ionizing radiation ("rate-dependance"), as well as long-term gain drifts over time. With concern for the impact such gain changes would have on the operation of a TPC, we investigated the gain stability of each of the detectors we built. These measurements involved three different types of GEM foils, curved and flat geometries, different readout electrode configurations, various transfer and induction field settings, and multiple generations of electronics. With all of these conditions being varied it is impossible to make meaningful quantitative comparisons between one configuration and another. Further, we have no means to control or measure the moisture $\left(\mathrm{H}_{2} \mathrm{O}\right)$ content of our gases. However, each of the various data sets is consistent with the following general observations.

- GEM gain does depend on the high-voltage history.

- Upon initial application of high voltage, the gain increases to its ultimate value with a time constant of from 5 to 30 minutes. 


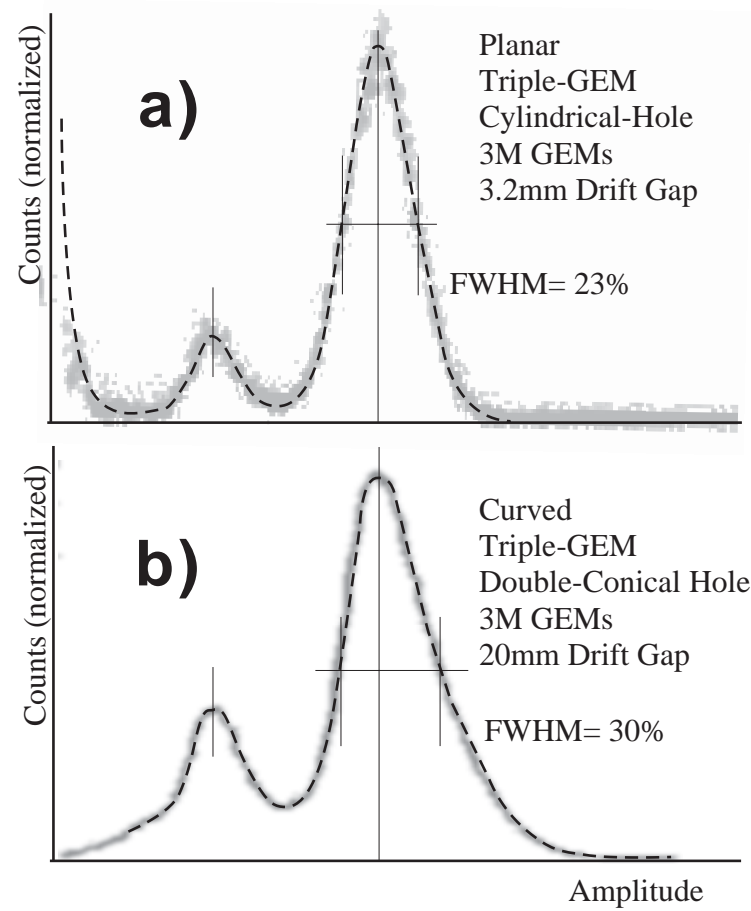

Figure 9. Pulse-height Spectra of ${ }^{55} \mathrm{Fe} \mathrm{X}$-rays obtained from a) Flat and b) Curved triple-GEM detectors filled with $80 / 20 \mathrm{ArCO}_{2}$. The two peaks correspond to the $5.9-\mathrm{keV}$ X-rays (main peak) and the argon escape peak $3.2 \mathrm{keV}$ below that. The planar detector contained a $3.2 \mathrm{~mm}$ drift gap, while the drift region of the cylindrical chamber was $20 \mathrm{~mm}$ deep radially.

- Upon restoration of high voltage after an interruption, the gain is reduced by an amount dependent upon the length of the interruption. It returns to near its previous value with a time constant similar to that observed upon initial turn-on. An interruption of one minute or less typically has a negligible impact.

- The long-term gain variation under constant high voltage and radiation flux is dominated by changes in atmospheric pressure.
- A 1000-fold increase in X-ray flux can cause as much as a $50 \%$ drop in gain. The gain recovers to its initial value if the flux is reduced to the previous lower value.

- The rate-induced drop in gain is observed only in the region of the detector receiving the high radiation flux. Other portions of the TPC are unaffected even though they share the same gas volume and highvoltage.

- The rate-dependence of gain is less when using 80/20 He/DME than when using $80 / 20 \mathrm{ArCO}_{2}$ (although this may merely reflect the lower cross section for X-rays in HeDME).

- The GEM shape (curved or flat) has no impact on any of the above behaviors.

The above observations lead us to include the follwing procedures when running the experiment and analyzing the data:

- The detector high voltage must be turned on at least 30 minutes prior to taking data

- Significant changes in beam intensity must be noted and the data must be monitored after such an event to determine when the detector operation has stbilized.

- The data must be monitored for needed adjustments of the gain calibration resulting from long-term drifts and/or atmospheric pressure variations.

We stress that all of these variations in gain with position, with rate, and with time, appear to be independent of whether the GEMs are flat or curved.

\section{The BoNuS RTPC}

The detector finally used for the BoNuS experiment was built upon the experience with fabrication techniques and operation of the prototypes discussed above. Here we describe its design and assembly, the results of pre-run testing, and its performance in the experiment. 


\subsection{Design}

As described in section 3.2, the detector was required to instrument a cylindrical annulus around the target and to fit within an existing magnet. To maximize acceptance and cause minimal scattering of secondary particles, mechanical components needed to be as small and lightweight as possible while maintaining a structure which would be rigid enough to support the GEMs, electrodes, and on-board electronics. The sensitive volume must begin just outside the region of high background particle flux near the target. It extends as far as the first GEM at $\mathrm{R}=60 \mathrm{~mm}$. The space outside the pad-board $(\mathrm{R}=69 \mathrm{~mm})$ was reserved for pre-amplifiers and cables.

The resulting device consists of two similar half-cylinder units which are mated together on either side of the central axis. Axial mechanical structures fit within $\pm 16^{\circ}$ wedges along the top and bottom of the assembly, as shown in Fig. 10. All of the structural components were machined out of Ultem ${ }^{\circledR}$. Each subassembly (window, cathode, three GEMs, and padboard) is self-supporting. These parts nest together to form the whole detector module. The interior walls of the drift region (two end caps and two vertical surfaces forming segments of a chord through the cylinder) are PCBs patterned with metal traces forming the field cage. The chord PCBs extend above and below the active portion of the detector and carry the high-voltage divider circuits and connection points, as well as pulseinjection circuitry for testing the electronics. Two vertical support plates, one above and one below the detector axis, form the backbone of the system. The other subassemblies are attached to either side of these plates. The detector is fixed to its external supporting structure by attachment to the upstream edges of these plates.

\subsection{Custom Gas Electron Multipliers}

The three GEM support frames were dimensioned such that identical GEM foils could be used throughout. These custom GEMs were manufactured by Tech-Etch[26], and have an active area covering $20 \mathrm{~cm} \times 17 \mathrm{~cm}$. The inner surface of each GEM is divided into four electrically isolated $5 \mathrm{~cm} \times 17 \mathrm{~cm}$ regions to reduce the energy stored in any single high-voltage element.

The GEMs were first cleaned and subjected to acceptance tests. They were rinsed with ethanol and blown dry with compressed nitrogen to remove the significant dust and contaminants. Then they were held by spring clips to temporary frames providing connections for bias voltage and were placed in a box flushed with dry nitrogen. Initially the GEMs were quite conductive, with side-to-side resistances as low as a few megohms per segment. After drying and conditioning with high-voltage for a few hours, the conductivity decreased. The foils were accepted if they achieved a leakage current of less than $1 \mu \mathrm{A}$ at 300 volts. About $90 \%$ of the GEMs passed this crude but effective test.

Before any of these GEMs were bonded to chamber components, a sample of them was tested in yet another prototype planar TPC to verify that they would meet the performance requirements. A set of two GEMs was assembled into this module with a $3 \mathrm{~mm}$ gap between them and between the final GEM and an electrode board. Tests with cosmic rays and radioactive sources demonstrated that the TechEtch GEMs could provide the required gain and stability for the RTPC when appropriate bias and transfer/induction electric fields were applied.

\subsection{Field Cage}

The electric field between two infinitely long concentric cylinders is perfectly radial with the magnitude at radius $r$ proportional to $r^{-1}$. To make the field within the drift volume of the BoNuS detector a close approximation to this, it was necessary to provide a field cage on the inner surfaces of the end caps and chord walls. Eleven discrete conductive arcs (on the end caps) and strips (on the chord walls) were held at the voltages that would be present at the center radius of each conductor in a perfectly radial field. ${ }^{3}$ The field cage conductor radii, widths, and relative voltages are listed in Table 1.

\footnotetext{
${ }^{3} \mathrm{~A}$ more elegant solution would have been to coat the end cap surfaces with a uniform resistive layer.
} 

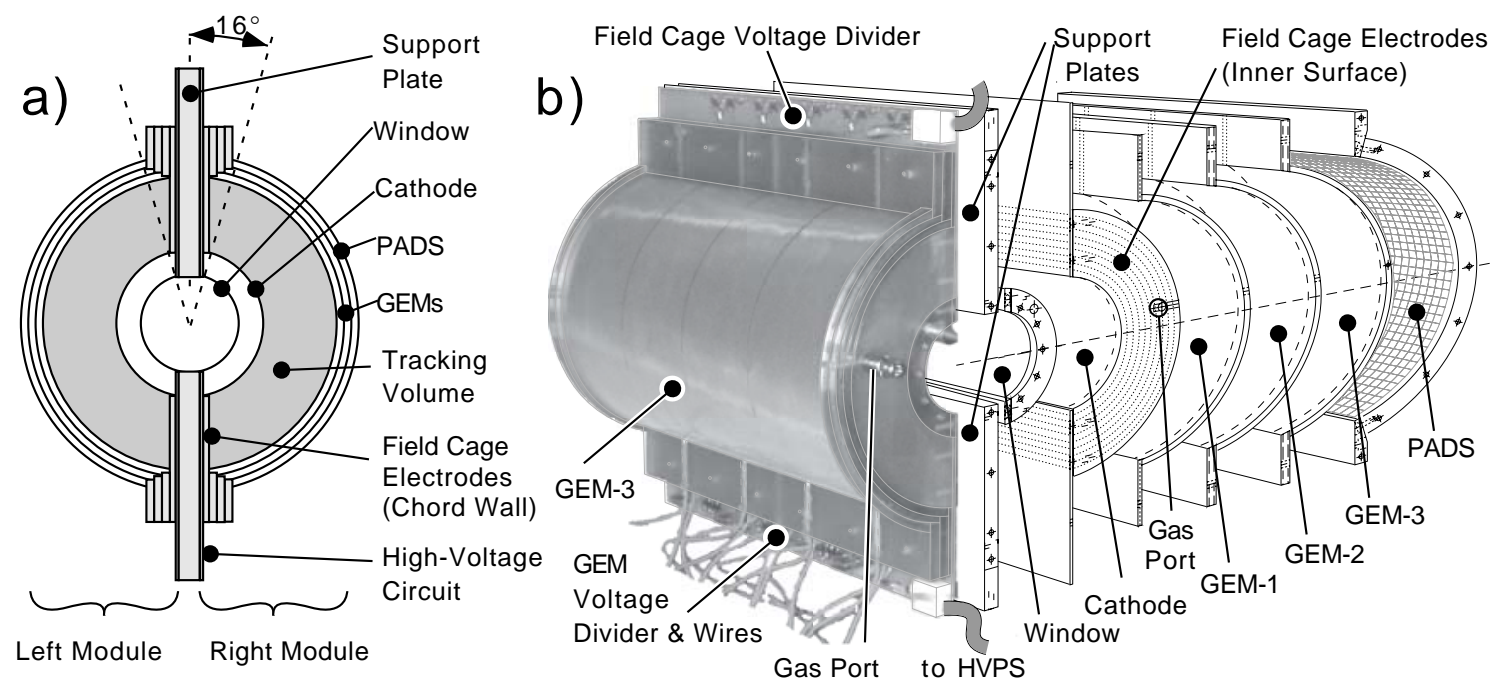

Figure 10. Photograph and Schematic Diagram of the BoNuS RTPC. a) Cross section view through the center of the detector. b) Photograph of the left module with the readout padboard removed and a complementary exploded view exposing the components of the right module.

\subsection{Attachment of Foils to the Frames}

The procedures for fabricating the full-size RTPC subassemblies were effectively the same as described in section 4.2 for the curved prototype, and will not be repeated here.

It is noteworthy that none of the sheet materials were held under tension when they were attached to their frames. Only the aluminized mylar has a modulus of elasticity that would allow the light-weight frames to support a noticeable tension without significant deformation. In particular, the GEMs were not stretched. The result is that any dimples present in the GEMs after manufacture and cleaning remain in the assembled unit. While none of the GEM surfaces were perfectly smooth, only the very poorest-quality foils had out-of-plane features with amplitudes approaching $1 \mathrm{~mm}$. None of the imperfections in the GEMs were linked with subsequent highvoltage breakdowns.

However, even the tiniest flexure (tens of $\mu \mathrm{m}$ ) of one of the frames will cause the attached sheet material to form a wrinkle. It was found that slight imperfections in the alignment fixtures or uneven clamping forces during assembly caused many of the subassemblies to show modest distortions. The aluminized mylar windows and cathodes were the most difficult to form, but the resulting $1 \mathrm{~mm}$ to $3 \mathrm{~mm}$ wrinkles are unimportant for these structures given the modest tracking resolution required of the detector. Similar imperfections in the GEM cylinders, however, resulted in catastrophic sparks between neighboring GEMs. Attempts to alleviate this problem by inserting up to three spacer arcs between the GEMs failed to eliminate the wrinkles. The spacers simply caused the wrinkles to move to different places, and sometimes made the wrinkle amplitudes larger. Only by carefully hand-fitting each shell of the detector module was it possible to achieve an assembly with proper spacing between layers. Clearly, a design using a monolithic structure that supported all three GEMs would have been a better choice.

\subsection{Analog Electronics}

The outermost layer of the detector is the padboard. The pattern of $4.45 \mathrm{~mm} \times 5 \mathrm{~mm}$ pads 
Table 1

Field-cage Conductor Locations, Widths, and Voltages (referenced to the cathode) for the case of a total drift-gap voltage of 1500 v.)

\begin{tabular}{cccc}
\hline $\begin{array}{c}\text { Strip } \\
\text { ID }\end{array}$ & $\begin{array}{c}\text { Center } \\
\text { Radius } \\
(\mathrm{mm})\end{array}$ & $\begin{array}{c}\text { Strip } \\
\text { Half-Width } \\
(\mathrm{mm})\end{array}$ & Voltage \\
\hline Cathode & 30.00 & 0.28 & 0 \\
2 & 32.72 & 0.67 & 188 \\
3 & 35.68 & 0.80 & 375 \\
4 & 38.91 & 0.94 & 563 \\
5 & 42.43 & 1.09 & 750 \\
6 & 46.27 & 0.75 & 938 \\
7 & 49.37 & 0.85 & 1078 \\
8 & 52.69 & 0.96 & 1219 \\
9 & 56.23 & 1.08 & 1359 \\
GEM-1 & 60.00 & 0.60 & 1500 \\
\hline
\end{tabular}

on the inner surface of this double-sided PCB is shown in Fig. 11. The board is composed of goldplated copper conductors on a glass fiber substrate, described earlier. Closed vias through the board connect each pad to a standard surfacemount connector pattern on the outer surface. The portion of the outside not occupied by a connector is plated to form a ground plane for electrical shielding.

Each connector carries sixteen pad signals and four ground connections and supports a preamplifier card. Two hundred cards are required to instrument the entire RTPC. A schematic for one channel of preamplifier (Inverter/Driver) is shown in Fig. 12. The circuit provides only modest gain $(-4 \mathrm{mV} / \mu \mathrm{A})$ and risetime $(\sim 50 \mathrm{~ns})$, and so is quite stable against oscillation. It is capable of driving the signals across a 6-m long, 0.635$\mathrm{mm}$ pitch ribbon cable to a low-impedance receiver circuit. Because the preamplifier uses lowpower components, it is possible to provide its supply voltages as well as sixteen ground and signal connections on each 34-conductor cable. Each 16-channel preamp card dissipates about $1 \mathrm{~W}$ of heat which is removed by air forced across the outside of the RTPC.

The preamplifier cards project radially from the surface of the detector and connect to the

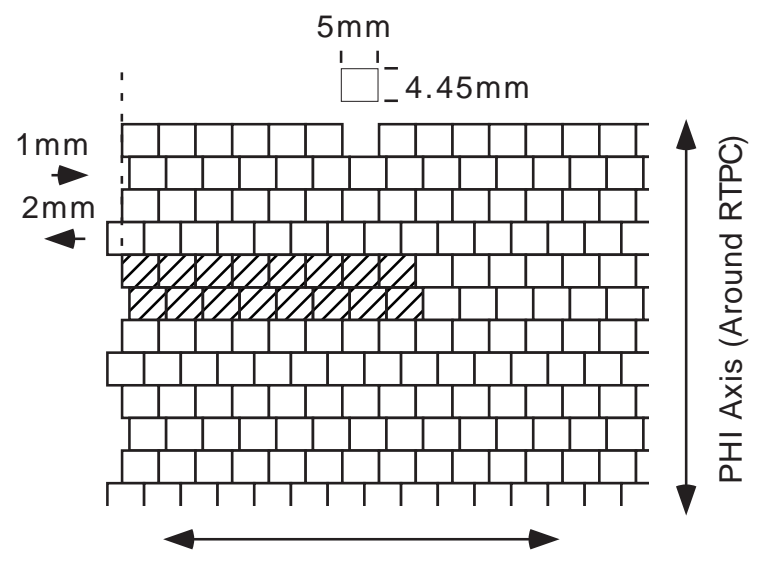

Z Axis (Parallel to Beamline)

Figure 11. Pad Geometry in the Production RTPC. There are 40 rows and 40 columns of pads. Pad rows (along cylindrical axis) are offset from one-another to improve the track resolution. Pads are connected to 16-channel preamp cards in groups similar to the shaded region of the figure.

ribbon cables in such a way that the cable length is parallel to the chamber axis. Carrying the preamplifier power on these cables and avoiding the need to fold them at the detector are features crucial to allowing the required electronics packing density.

Signals are received at the far end of the ribbons by buffer amplifiers built on 128-channel $23 \mathrm{~cm} \times 33 \mathrm{~cm}$ PCBs (the receiver cards). The schematic of this circuit is also shown in Fig. 12. The designs of the preamps and receivers were chosen to minimize crosstalk and sensitivity to external electronic noise. The network following the receiver stage conditions the signals so that they are compatible with the inputs of the preamplifier / shaping amplifier (PASA) of the ALTRO[29] Front End Cards (FEC) used for digitization and readout. The PASAs had been designed to attach directly to the end caps of the the ALICE TPC[30], but the compact geometry and signal polarity of BoNuS would not allow a similar implementation. 


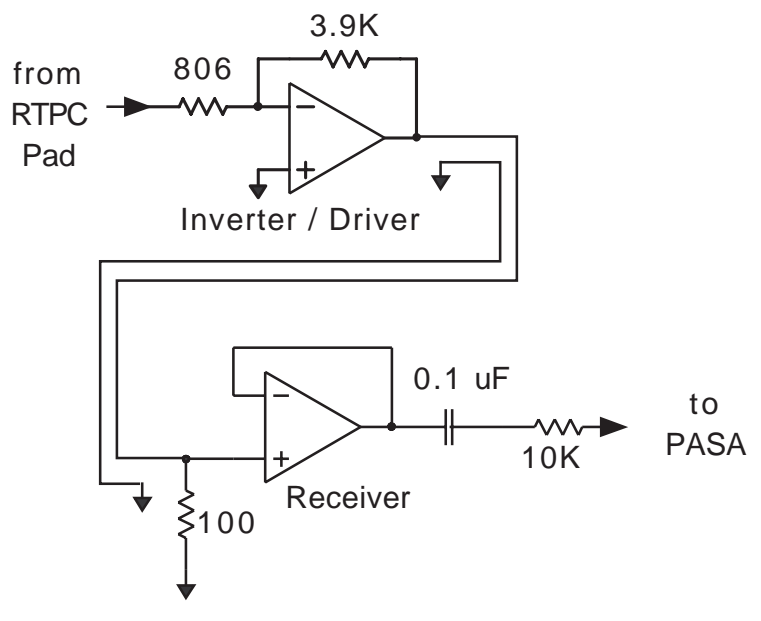

Figure 12. Preamplifier and Receiver Schematic. Between these two stages is a $\sim 6 \mathrm{~m}$ long ribbon cable. The OP-AMP chips are Texas Instruments OPA4354[28].

\subsection{Readout}

In addition to the receiver circuits, each receiver card carries a standard ALTRO FEC as a daughter card. Thirteen receiver boards with FECs are housed in each of two standard crates meeting VME mechanical standards. The backplanes in these crates were custom designed to provide the control and data busses required by the ALTRO system. An ALTRO readout controller (U2F) supervises the backplane communications in each crate and transfers the compacted digital data to a pair of single-board (VME) computers via USB-2 interfaces. These processors serve as Readout Controllers (ROC's) within the standard CLAS data acquisition system. As configured, the system provides readout of $\sim 1 \mathrm{kB}$ events at a rate of about $500 \mathrm{~Hz}$. Because the readout rate is a function of the event size, it is important to keep noise and oscillations to a minimum. Enabling of the multi-buffering scheme available in the ALTRO system would allow higher rates.

BoNuS event readout was initiated by the standard CLAS electron trigger system, selecting in- teractions with a high probability of having an electron track in CLAS. The data recorded for each event is composed of the times (114 ns samples) and amplitudes (10 bits) of all TPC pad signals above threshold for a time period extending from $1.7 \mu$ s before to $9.7 \mu$ s after a trigger. This interval is about 1.5 times the maximum drift time in the RTPC.

In order to verify synchronization between the ALTRO and CLAS data acquisition systems, special triggers were sent simultaneously to both systems about once each second. These triggers were flagged by a logic signal presented to both systems in time with the trigger. Recording such a flag is a standard function of the Trigger Interface modules used by CLAS. For the ALTRO system, the logic pulse was electronically modified so that it could be injected as analog data directly into one of the unused ALTRO channels. By verifying that both systems reported the flag pulses in the same events it is possible to guarantee that they were operating synchronously.

\section{Operation of the Detector}

\subsection{Running Conditions \\ 6.1.1. Gas}

The gas chosen for operating the detector is a mixture of high-purity helium and dimethylether (DME), mixed in the ratio $4: 1$ by volume. Using helium as the primary component provides a lowdensity medium, required for minimizing the energy loss of the slow protons. The He/DME mixture provides stable performance and sufficient gain in the GEMs at a relatively low bias voltage (as compared to $\mathrm{ArCO}_{2}$, for example). It also exhibits an acceptable Lorentz angle, from $34^{\circ}$ to $38^{\circ}$ in the electric and magnetic fields present in this detector. To further reduce energy lost by the particles, the volume between the target straw and the entrance window of the RTPC was continuously flushed with helium.

\subsubsection{Gain Settings}

Optimal measurement of the ionization energy loss of the spectator protons requires the highest possible gain (maximum signal to noise ratio) that maintains the largest signals below that 
amplitude at which electronic nonlinearities begin to occur. The most heavily-ionizing particles present in the experiment in significant number are the spectator protons. Tests had shown that the interaction between the receiver circuit and the PASA inputs leads to nonlinear response when the digitized signals exceed $50 \%$ of fullscale in the ALTRO digitizers. To determine the optimal high-voltage setpoints in the RTPC, runs were taken covering a range of bias voltages with the high-energy electron beam striking the deuterium-filled target. The highest setting at which fewer than $1 \%$ of the charge samples exceeded 512 ADC units was noted and used for production running.

Various calibration runs were needed in which the particles to be tracked in the RTPC were less heavily ionizing. The bias setting for these runs was determined using similar criteria when the target cell was filled with hydrogen. Thus the gain was set for maximum non-saturated signals from elastically scattered protons.

For all runs, the magnitude of the (negative) cathode power supply voltage was maintained $1500 \mathrm{~V}$ higher than the GEM supply voltage, establishing the field in the drift region of the RTPC. The fields produced by this setting provided a sufficiently short clearing time in the drift region without making the cathode voltage so high that breakdowns would occur. Table 2 summarizes the resulting voltages applied to all elements of the detector during the experiment. Note that the presence of filter and current-limiting resistors causes the GEM and cathode voltages to be lower than the supply voltages.

\subsubsection{Electronic Threshold and Data Compression}

The ALTRO system provides extensive datacompression capabilities. Channels with signals never exceeding a threshold determined just prior to each event are completely suppressed during data transfer. With the detector configured for tracking spectator protons, this typically eliminated the readout of about $99 \%$ of the channels. Further compaction of the data was achieved by suppressing the readout of samples that remained
Table 2

Supply Settings and Electrode Voltages in the RTPC during operation of the experiment. The suffixes on the GEM labels refer to the inner (i) and outer (o) surfaces of the GEMs. All voltages are of negative polarity and are referenced to ground.

\begin{tabular}{|c|c|c|c|c|}
\hline \multirow[t]{3}{*}{$\begin{array}{l}\text { Detector } \\
\text { Element }\end{array}$} & \multicolumn{2}{|c|}{$\begin{array}{c}\text { Heavily } \\
\text { Ionizing } \\
\text { Tracks }\end{array}$} & \multicolumn{2}{|c|}{$\begin{array}{c}\text { Minimum } \\
\text { Ionizing } \\
\text { Tracks }\end{array}$} \\
\hline & Left & Right & Left & Right \\
\hline & Half & Half & Half & Half \\
\hline HVPS-C & 4550 & 4350 & 4950 & 4750 \\
\hline HVPS-G & 3050 & 2850 & 3450 & 3250 \\
\hline Window & 0 & 0 & 0 & 0 \\
\hline Cathode & 4532 & 4329 & 4931 & 4729 \\
\hline GEM-1i & 2869 & 2656 & 3242 & 3026 \\
\hline GEM-1o & 2579 & 2374 & 2915 & 2705 \\
\hline GEM-2i & 2087 & 1918 & 2359 & 2185 \\
\hline GEM-2o & 1798 & 1642 & 2031 & 1871 \\
\hline GEM-3i & 1143 & 1040 & 1292 & 1185 \\
\hline GEM-3o & 845 & 764 & 955 & 871 \\
\hline Padboard & 0 & 0 & 0 & 0 \\
\hline
\end{tabular}

within 5 counts of each channel's moving average baseline. Although the readout of a single channel represents 100 samples taken by a 10-bit ADC, after all compression the typical BoNuS event contained only about 1000 bytes of ALTRO data.

\subsection{Analysis and Detector Calibration 6.2.1. Tracking}

Using even a very basic drift-velocity calibration tracks are readily apparant in the detector. Fig. 13 shows various views of one typical event with a spectator proton originating near the downstream end of the BoNuS RTPC. The high density of hits along the tracks make even simple pattern-recognition algorithms efficient at finding tracks. The method used links hits together if they are pairwise near one-another in space. A helix, constrained to pass through the beamline, is fitted to the group of chosen points. The line tangent to the helix as it crosses the beamline is taken as the initial direction vector for the track. The particle's momentum and charge can be cal- 
culated from the track curvature, polar angle, and the magnetic field. These results may be used as the starting point for a more refined fitting procedure which accounts for energy loss and the nonuniform magnetic field. In combination with the measured momentum, energy-loss $(d E / d x)$ measurements derived from the signal pulse heights can be used to provide particle identification.

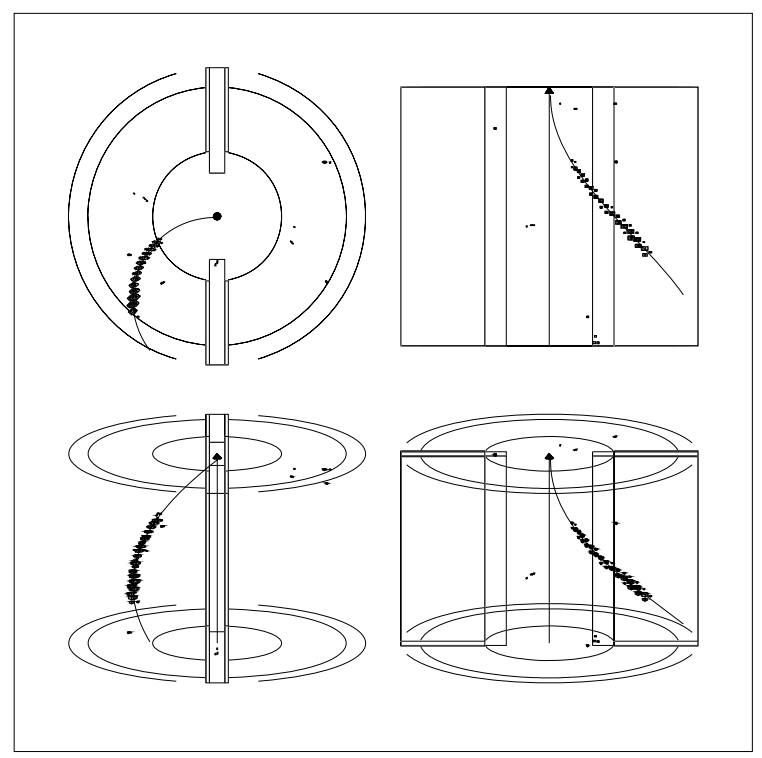

Figure 13. Online Display of RTPC Data representing a Spectator Proton. Individual charge clusters are displayed as boxes, centered at the point of origin of each cluster. The box size represents the measured pulse height. When viewing along the beam line (top-left), one can see the track curvature. The polar emission angle is apparent in the side view (top-right), and the perspective views (bottom).

\subsubsection{Drift Velocity Calibration}

To make use of the data from any TPC it is necessary to know the trajectories of ionization electrons as they drift from through the gas to the GEMs and to the readout electrodes. Given that the electric field in a radial TPC is not constant, and that the magnetic field in the BoNuS RTPC is both varying and almost orthogonal to the electric field, the drift velocity calculation for this experiment is not straightforward to solve analytically. The approach we have taken is to use the program MAGBOLTZ[31] to generate approximations to the electron paths.

Using a parametrization of the electric and magnetic fields, and the approximate composition of the gas, MAGBOLTZ predicted the path taken by electrons that terminate at the center of each pad. These results were used to create a function that converts an observed signal into a space point $P$ :

$$
P_{x y z}=P_{x y z}\left(I, T_{\text {sig }} ; V_{\text {cathode }}, V_{G E M}, R_{\text {gas }}\right),
$$

where $I$ is the pad number and $T_{s i g}$ is the time (in ALTRO units) at which a signal was recorded. The parameters $V_{\text {cathode }}$ and $V_{G E M}$ represent the RTPC operating voltages, and the fraction of helium in the gas mixture is given by $R_{\text {gas }}$. Idealy, the drift paths determined this way would be final. However, the high magnetic field and the imperfect knowledge of the gas mixture led us to include the three parameters and determine effective values for them that provided the best agreement with the data.

Limited resources and schedule contstraints did not allow inclusion of a calibration-track generator (laser or charge-injection system), so the tracking calibration was performed using physics data. Particle tracks measured by CLAS were projected back through the RTPC. Hits in the RTPC were associated with those tracks using the approximate drift-path parameterization. Finally, the function minimization package MINUIT was used to find values for the drift-path parameters that yielded the best overall agreement between the reconstructed RTPC hit positions and the tracks reconstructed by CLAS. Fig. 14 shows a representative result of this procedure: .

This fitting procedure could only be applied to those special calibration runs taken with the RTPC gain set unusually high so that it was sensitive to minimum-ionizing particles. The larger fraction of the data was taken with the gain 


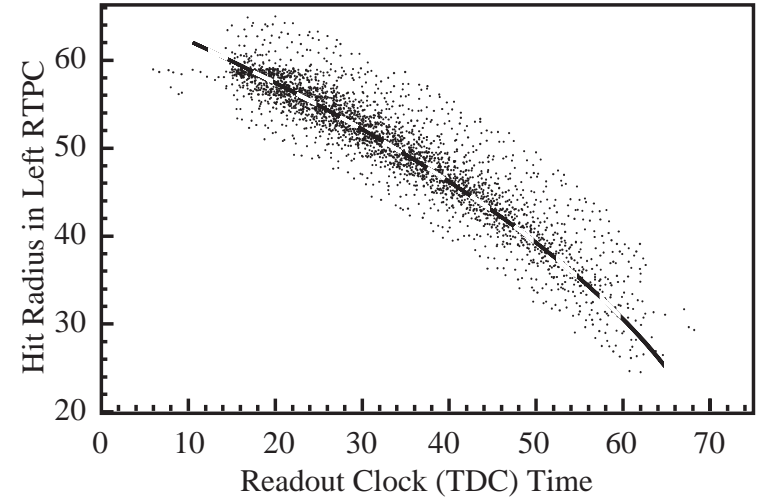

Figure 14. Drift Path Calibration. Each entry in the scatter plot indicates the radial coordinate $(R)$ and ALTRO-time $(t)$ of a hit in the RTPC, where the radius is given by the point where the CLAS track intersects the $Z$ - plane containing the readout pad on which the signal occured. The heavy dashed line indicates the final parametrization of the function $R(t)$.

set about ten times lower so that only heavilyionizing tracks could be reconstructed in the RTPC. However, even at this low gain setting, minimum-ionizing particles occasionally deposit detectable clusters of charge. By searching for RTPC hits along the trajectories of such particles it was possible to to obtain assurance that the fitted drift paths are consistent with the observed hit locations, even though the hits are too sparse to allow an independent determination of the drift paths.

\subsubsection{Gain Calibration}

The entire electronics chain was bench-tested prior to installation. No channels were found whose response to a test pulse was more than a few percent away from the mean response. As the tracking analysis matured, however, it became apparent that the effective detector gain varied significantly across the surface of the RTPC. Therefore it was necessary to develop a procedure for determining the relative responses of all 3200 pads before useful $\mathrm{dE} / \mathrm{dx}$ information could be extracted from the data.

A self-calibration was performed using heavilyionizing tracks obtained from the RTPC data alone. Making use of the electron drift paths determined earlier, each track's momentum was determined. The momentum was used in the Bethe-Bloch formula to find the average $\mathrm{dE} / \mathrm{dx}$ expected for a proton along each segment of the track. Again using the drift paths, the amount of energy-loss whose ionization electrons should have drifted onto each readout pad $\left(E_{i, j}\right)$ was calculated. For each track (i) and pad (j), the following mean response ratio was computed

$$
G 1_{j}=\sum_{i=1}^{N}\left(Q_{i, j} / E_{i, j}\right) / N,
$$

where the sums over $i$ for each pad run over only those track segments that were predicted to produce a signal in pad $j . Q_{i, j}$ is time-integrated pulse-height measured on the pad for the given track. Although not all of the tracks were created by protons, this mis-assignment causes an error only in the overall scale of the gain estimate, as the mixture of particle species throughout the RTPC appears to be reasonably uniform. These first-pass gain-normalization factors, $G 1_{j}$, were used to scale the raw pulse-heights and the same calculation was performed again but excluding tracks whose measured $d E / d x$ was inconsistent with that expected for protons. These secondpass gain- normalization factors (which are only slightly different than the $G 1_{j}$ ) are retained and used for the final physics analysis particle identification.

The relative gains are shown in Fig. 15, where the darkness of each element indicates the response of the corresponding pad. Nonfunctioning channels (failed wiring or amplifier, etc.) are indicated by white. Some regional variations in triple-GEM gain can be seen, such as the dark diagonal band near the center of row 70 , and the forward portion of the left-hand module (bottom half of the figure). The diagnoal band is probably the result of a wrinkle remaining in one of the GEMs, while the broader variation in the left module probably was caused by nonuniformity of the GEM itself. 


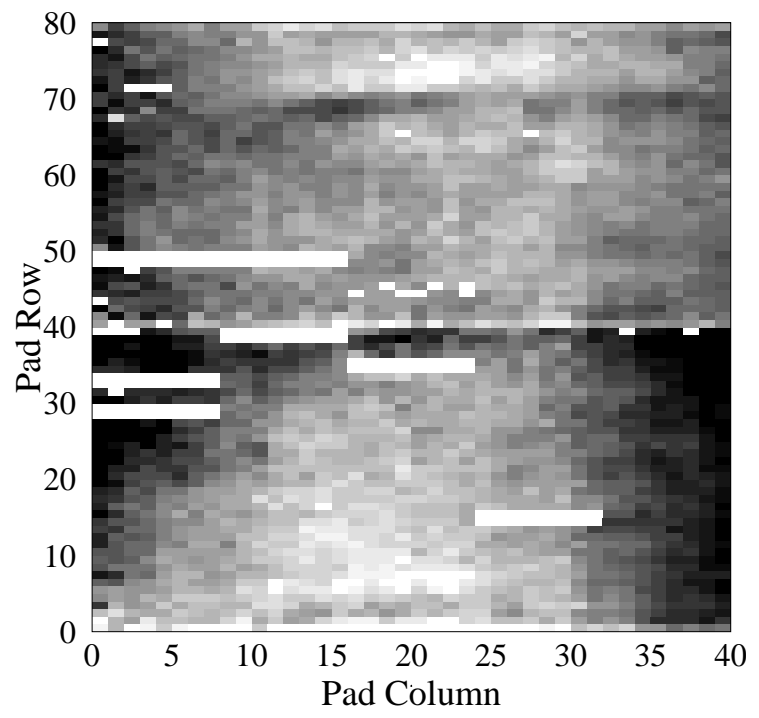

Figure 15. Relative gains determined for each pad across the surface of the RTPC. Each box represents one pad. The horizontal axis is the pad column number along the RTPC length $(Z)$, and the vertical axis is the pad row number $(\Phi$ coordinate). Darker grey cells have higher gain, while white cells were not functioning. In this representation, the greyscale is logarithmic from 0.6 to 6.0 , a range which covers about $95 \%$ of the measured gains.

\section{Performance}

The performance of the BONUS RTPC is summarized in the figures below. Differences between the event vertices determined by the RTPC and, independently, by the CLAS detectors is shown in Fig. 16. Only very loose track-quality cuts were applied in generating this distribution. Agreement between the two tracking systems is seen to be good. The resulting $Z_{\text {vertex }}$ difference distribution has a width of about $5 \mathrm{~mm}$, consistent with the resolution provided by CLAS alone.

Scattering angle differences measured by comparing elastically scattered electrons that were tracked in both the RTPC and in CLAS are

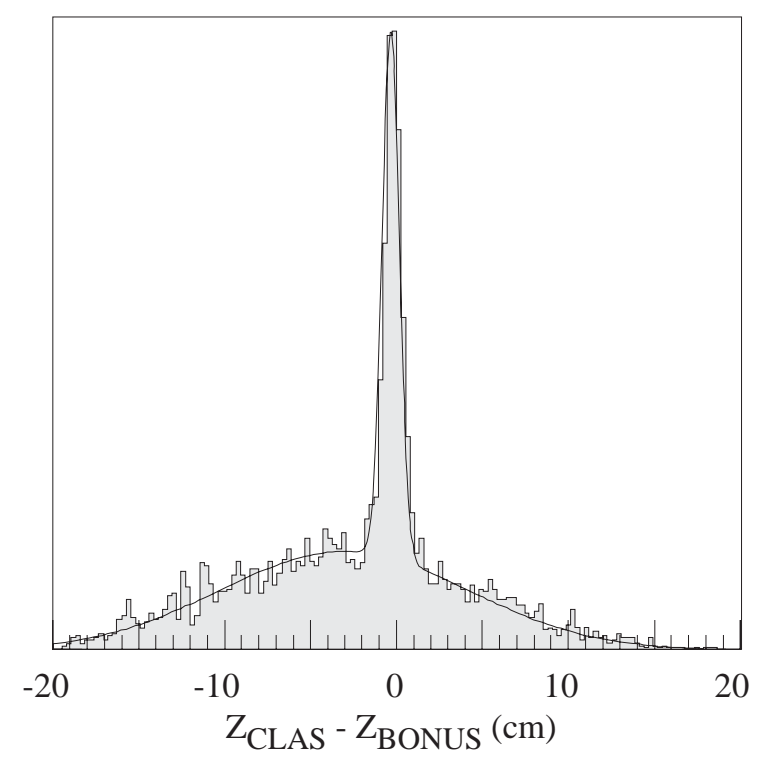

Figure 16. Difference between the Positions of Interaction Vertices measured by the RTPC and by the CLAS. The curve shows a two-Gaussian fit to the data. The width of the narrow peak is $\sigma=5 \mathrm{~mm}$.

shown in Fig. 17. These electrons probe the most forward portions of the RTPC acceptance. The same comparison for elastic protons tracked by the RTPC is shown in Fig. 18, where in this case the CLAS prediction for the proton scattering angle comes not from direct measurement of the proton, but from measurement of the electron and a calculation which assumes that the ep scatter was elastic. Elastically scattered protons that are visible in the RTPC are scattered at $80^{\circ}$ to $90^{\circ}$. The widths of both distributions are consistent with the resolution expected of the RTPC given its geometry and readout electrode dimensions.

Given the RTPC's small size and somewhat course space-point resolution $\left(\sigma_{R}=\right.$ $0.53 \mathrm{~mm}, \sigma_{\phi}=0.025, \sigma_{Z}=1.2 \mathrm{~mm}$ ), the track momentum determined from curvature in the magnetic field is not expected to be meaningful above about $200 \mathrm{MeV} / \mathrm{c}$. Elastically scattered protons in our data sample all have momenta 


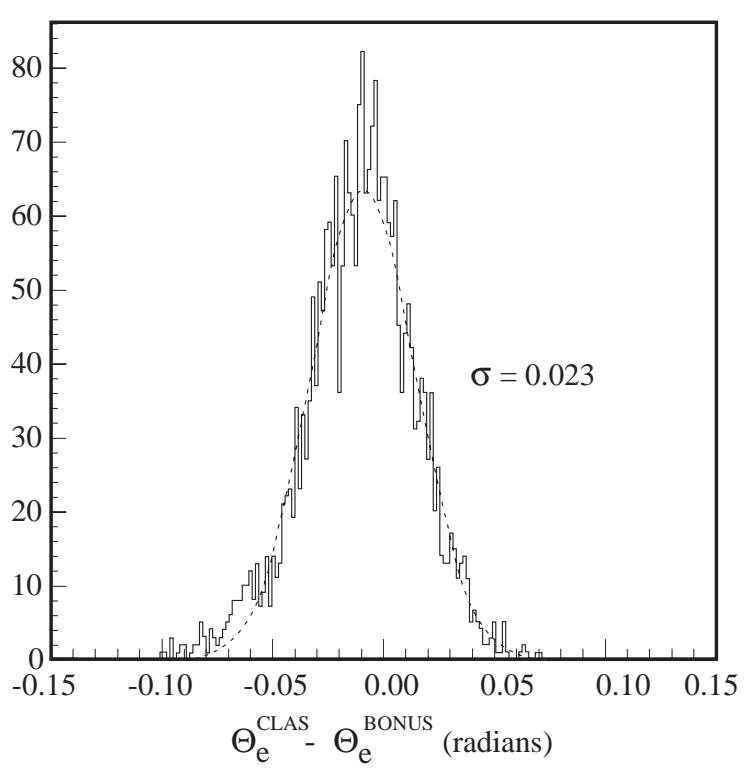

Figure 17. Difference between measurements of the electron scattering angle by CLAS and by the BoNuS RTPC.

above this limit and provide no measure of the RTPC's momentum resolution for the relatively slow spectator protons of interest. Evidence that the measured momenta are useful is provided by the following two analyses, however.

The mass spectrum of the recoiling object in quasielastic $e d \rightarrow e^{\prime} X p_{s}$ events computed both with and without using the measured spectator proton momentum is shown in Fig. 19. One expects to see peaks for the ground-state neutron as well as neutron resonances. The figure shows a significant improvement in the recoil mass resolution when the spectator proton momentum measured by the RTPC is included in the calculation.

The second momentum analysis also demonstrates the energy-loss $(d E / d x)$ sensitivity of the detetor. Data was taken while the target was filled with ${ }^{4} \mathrm{He}$ gas. Fig. 20 shows the correlation between observed ionization densities and measured track momenta for these events. Two bands are clearly seen. The lower narrow band is consis-

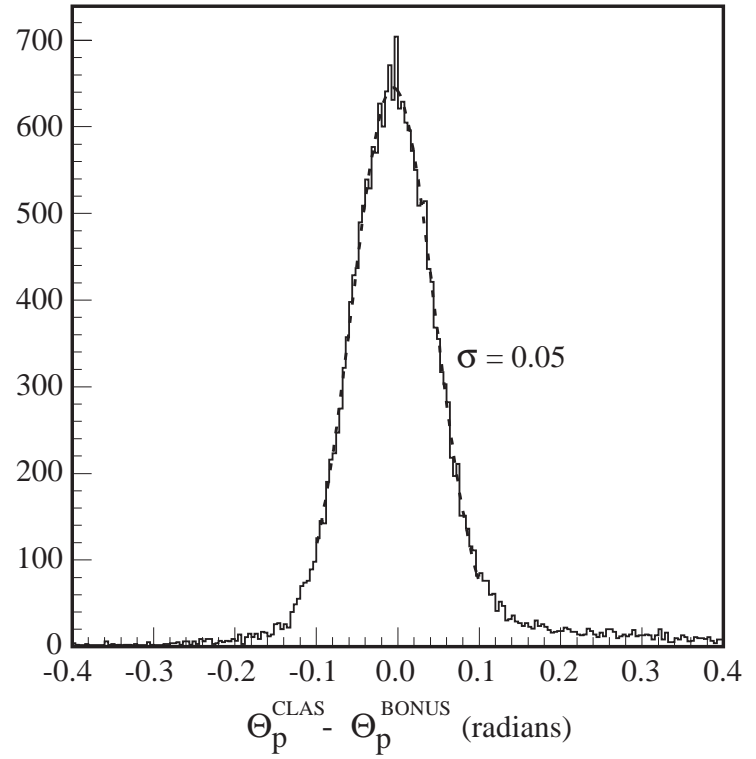

Figure 18. Difference between the measured and predicted proton scattering angle in ep elastic events (see text).

tent with tracks left by protons, while the more diffuse band corresponds to ${ }^{3} \mathrm{H}$ and ${ }^{3} \mathrm{He}$ tracks. The curve superposed on this scatter-plot indicates the mean energy-loss vs. track momentum predicted by the Bethe-Bloch formula for protons in $80 / 20 \mathrm{He} / \mathrm{DME}$.

\section{Summary}

A radial time projection chamber using a tripleGEM gain stage was successfully developed and was operated for two months during the run of a nuclear physics experiment. The GEMs are curved to conform to the cylindrical shape of the outer surface of the TPC. Fabrication of the curved GEM structures required careful assembly, but, as predicted, there were no fundamental difficulties associated with operating GEMs in a non-planar configuration.

Data resulting from the experiment is presently being analyzed to provide new information about the structure of neutrons, closer to what has 


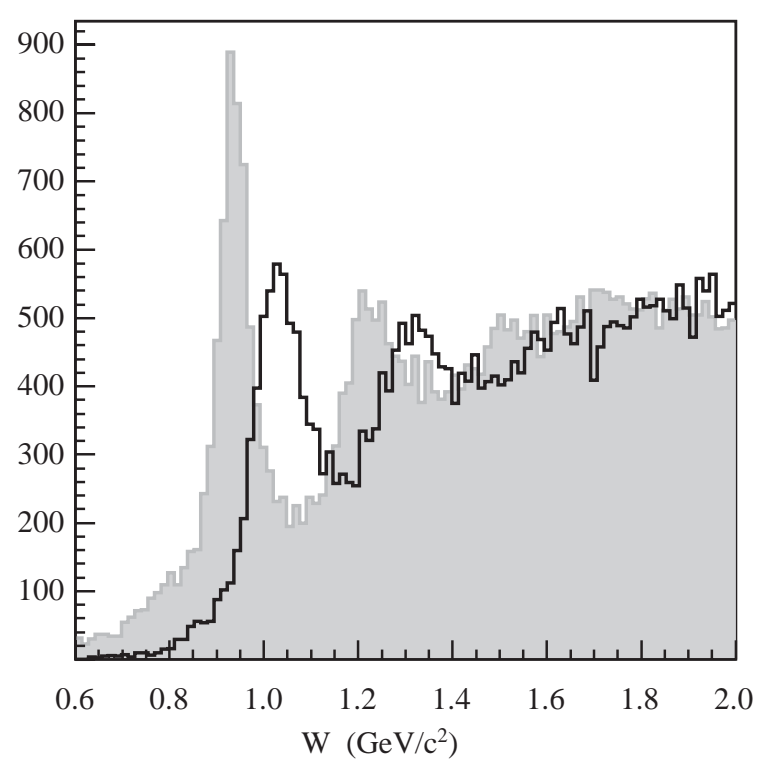

Figure 19. Recoil Mass Spectra for Quasielastic $e d \rightarrow e^{\prime} X p_{s}$ Events. The grey histogram shows the mass distribution computed when the spectator proton momentum vector measured in the RTPC is included in the calculation. The black line indicates the spectrum obtained when the spectator proton momentum is ignored.

been learned about protons in previous scattering experiments. RTPC data in particular are yielding to careful analysis and calibration, and indicate that the fundamental cylindrical GEM RTPC technique is quite useful and robust.

\section{REFERENCES}

1. F. Sauli, Nucl. Instr. and Meth. A 386 (1997) 531; R. Bouclier, et al., IEEE Trans. Nucl. Sci. 44(1997) 646.

2. F. Sauli, GEM readout of the Time Projection Chamber, CERN-EP-TA1 Internal report, July 29,1999.

3. B. Yu, et al., IEEE Trans. Nucl. Sci. 50 (2003) 836 ;

4. D. Karlen, et al., IEEE Nucl. Sci. Symp. Conf. Record (2003) 535.

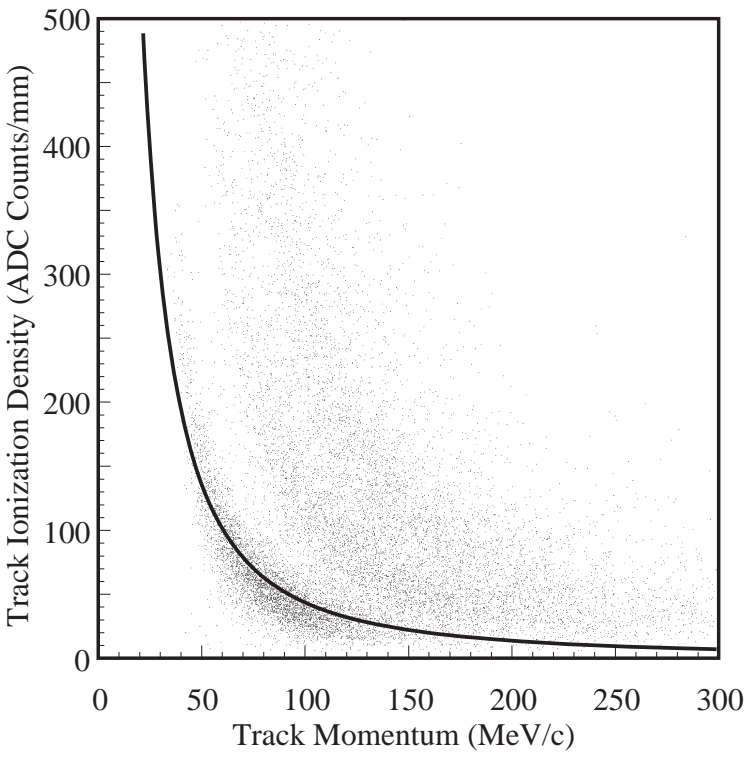

Figure 20. Track Ionization Density vs. Momentum. Both quantities come from measurements in the RTPC. The lowest band is consistent with the $d E / d x$ expected for protons. The diffuse intermediate band appears to come from deuterons, while events in the higher region are consistent with ${ }^{3} \mathrm{H}$ and ${ }^{3} \mathrm{He}$ nuclei. The curve shows the prediction of the Bethe-Bloch formula for protons.

5. J. Benlloch, et al., IEEE Trans. Nucl. Sci. 45 (1998) 234; F. Sauli, Nucl. Instr. and Meth. A 422 (1999) 257.

6. I. Niculescu et al., Phys. Rev. Lett. 85 (2000) 1186; I. Niculescu et al., Phys. Rev. Lett. 85 (2000) 1182; R. Ent, C.E. Keppel, and I. Niculescu, Phys. Rev. D 62 (200) 073008; S. Liuti, R. Ent, C.E. Keppel and I. Niculescu, hep-ph/0111063.

7. L.W. Whitlow et al., Phys. Lett. B282 (1992) 475.

8. P. Amaudruz et al., Phys. Lett. B295 (1992) 159.

9. T. Kitagaki, et al., Phys. Rev. D 42 (1990) 1331; A. Bergier, et al., Z. Phys. C 5 (1980) (265), and references therein.

10. J. R. Dunning et al., Phys. Rev. 141 (1966) 
1286; R. Bilger, et al., Nucl. Inst. and Meth. A 457 (2001) 64; I. Lehmann, et al., Nucl. Instr. and Meth. A 530 (2004) 275; A. Klimenko, et al., Phys. Rev. C 73 (2006) 035212.

11. G. Dodge, et al., "The Structure of the Free Neutron Via Spectator Tagging" Proposal to Jefferson Lab PAC23 (2003) http://www.jlab.org/

12. B. A. Mecking, et al., Nucl. Instr. and Meth., A 503 (2003) 513.

13. R. Cahn and G. Trilling, Phys. Rev. D 69 (2004) 011501.

14. D. Daughty, et al., "Deeply Virtual Compton Scattering with CLAS at 6 GeV" Proposal to Jefferson Lab PAC20 (2001) http://www.jlab.org/

15. DuPont High Performance Materials, Circleville, OH, USA

16. K.H. Ackermann, et al., Nucl. Instr. and Meth. A 499 (2003) 713

17. J. Kaminski, et al., Test Beam Studies of a GEM-Based Time Projection Chamber, S. Roth, et al., Design and Construction of a TPC Using GEM Foils for Gas Amplification, M. Ronan, et al., Precision Measurements of Diffusion and Resolution for a Large-Area Micromegas Time Projection Chamber, and M. Dixit, et al., Resolution of a MPGD Readout TPC Using the Charge Dispersion Signal, all in IEEE Nucl. Sci. Symp. Conf. Record (2004).

18. F. Sauli, 2002, private communication.

19. 3M Microinterconnect Systems Division, 6901 Riverplace Blvd., Austin, TX, USA

20. B. Ketzer, et al.,IEEE Trans. on Nucl. Sci.49 (2002).

21. Ohmite Mfg. Co., 1600 Golf Rd., Rolling Meadows, IL, USA

22. LeCroy Research Systems, Chestnut Ridge, NY, USA

23. Triangle Universities Nuclear Laboratory, Durham, NC, USA

24. General Electric Company, One Plastics Avenue, Pittsfield, MA, USA

25. Tra-con, Inc., Bedford, MA, USA

26. Tech-Etch, Inc., 45 Aldrin Road, Plymouth, MA, USA

27. R. Bouclier et al., Nucl. Inst. and Meth, A
396 (1997) 50.

28. Texas Instruments, Inc., Dallas, TX, USA

29. L. Musa et al., IEEE Nucl. Sci. Symp. Conf. Record (2003) 3647; R. Esteve Bosch et al., IEEE Trans. on Nucl. Sci. 50 (2003) 2460.

30. L. Musa, "The Time Projection Chamber for the ALICE Experiment", Nucl. Phys. A 715 (2003) 843;

31. S. Biagi, MAGBOLTZ programme, version 2, CERNLIB, CERN, Geneva, Switzerland (2005). 